\title{
Von der Präsenz- zur Hybridveranstaltung. Erfahrungen mit der Transformation einer Konferenzreihe zur Online-Netzwerkforschung.
}

\author{
T. Köhler ${ }^{1}$, E. Schoop ${ }^{2}$, N. Filz ${ }^{3}$, N. Kahnwald ${ }^{4}$, R. Sonntag ${ }^{5}$ \\ ${ }^{1}$ Professur Bildungstechnologie, Fakultät Erziehungswissenschaften, TU Dresden \\ ${ }^{2}$ Professur Wirtschaftsinformatik - Informationsmanagement, Fakultät Wirtschaftswissenschaften, TU Dresden \\ ${ }^{3}$ Medienzentrum, TU Dresden \\ ${ }^{4}$ Professur Wissens- und Informationsmanagement, Hochschule der DGUV (HGU), Bad Hersfeld \\ ${ }^{5}$ Professur Marketing, insbesondere Multimedia-Marketing, Fakultät Wirtschaftswissenschaften, HTW Dresden
}

\begin{abstract}
GeNeMe steht seit mehr als 20 Jahren für "Gemeinschaften in Neuen Medien“ und behandelt Online Communities an der Schnittstelle zwischen bzw. aus Sicht mehrerer Fachdisziplinen wie Informatik, Medientechnologie, Wirtschaftswissenschaft, Bildungs- und Informationswissenschaft sowie Sozial- und Kommunikationswissenschaft. 2020 haben sich die Autor:innen in ihrer Funktion als Ausrichter der Konferenz bewusst für den Fokus des Transfers bzw. Zusammenspiels von hybriden Realitäten zu hybriden Gemeinschaften entschieden. Überraschenderweise führte dies zu einem sprunghaften Anstieg der Beitragseinreichungen um ca. $80 \%$.

Der Aufsatz beschreibt, welche Erfahrungen mit der Transformation der Konferenzreihe zur Online-Netzwerkforschung, ausgerichtet durch mehrere Hochschulen am (virtuell-hybriden) Standort Dresden, die Autor:innen dabei gesammelt haben und macht Vorschläge, wie diese für die akademische Lehre verallgemeinert werden können.
\end{abstract}

GeNeMe has stood for "Communities in New Media" for more than 20 years and deals with online communities at the interface between or from the perspective of several disciplines such as computer science, media technology, economics, education and information science as well as social and communication science. 2020, the authors, in their capacity as conference organisers, deliberately chose to focus on the transfer or interplay of hybrid realities to hybrid communities. Surprisingly, this led to a jump in paper submissions of about $80 \%$.

The paper describes the experience the authors have gained with the transformation of the conference series on online network research, hosted by several universities at the (virtual-hybrid) location of Dresden, and makes suggestions on how this can be generalised for academic teaching.

*Corresponding author: Thomas.Koehler@tu-dresden.de 


\section{Generelles}

GeNeMe ${ }^{1}$ steht seit mehr als 20 Jahren für "Gemeinschaften in Neuen Medien" und behandelt Online Communities an der Schnittstelle zwischen bzw. aus Sicht mehrerer Fachdisziplinen wie Informatik, Medientechnologie, Wirtschaftswissenschaft, Bildungs- und Informationswissenschaft sowie Sozial- und Kommunikationswissenschaft. Als das Forum für den interdisziplinären Dialog zwischen Wissenschaft, Wirtschaft und Verwaltung ermöglicht die Tagung seit inzwischen 23 Jahren den Erfahrungs- und Wissensaustausch zwischen Teilnehmenden verschiedenster Fachrichtungen, Organisationen und Institutionen. Einen umfassenden thematischen Überblick über die Konferenzthemen und deren Veränderung über die Zeit geben Köhler et al. [1].

Nicht erst seit der Pandemie, sondern seit Beginn der Konferenzserie 1998 geht es kontinuierlich um die wissenschaftliche und anwendungsbezogene Analyse von mediengestützter Kooperation. Einen Schwerpunkt bilden immer wieder die Auseinandersetzung und auch Erprobung bildungsbezogener Formate der Wissenskooperation. 2020 haben sich die Ausrichter:innen bewusst für den Fokus des Transfers bzw. Zusammenspiels von hybriden Realitäten zu hybriden Gemeinschaften entschieden. Überraschenderweise führte dies zu einem sprunghaften Anstieg der Beitragseinreichungen auf um ca. 80 \% auf ca. 90 Beiträge - wobei die Kausalität unklar ist.

Im Folgenden wollen wir untersuchen, welche Erfahrungen mit der Transformation der Konferenzreihe zur Online-Netzwerkforschung, ausgerichtet durch mehrere Hochschulen am (virtuell-hybriden) Standort Dresden, die Autor:innen dabei gesammelt haben. Damit ist der Beitrag anschlussfähig zu aktuellen Studien rund um Möglichkeiten digital gestützter, hochschulübergreifender Kooperation in der Lehre. [2], [3]

\section{Tagungsablauf, -ort und Programminformation}

Das Programm der GeNeMe 2020 [4] war vielfältig aufgestellt, umfassend eine eintägige Vorkonferenz und eine zweitägige Hauptkonferenz.

Für die Visualisierung der Programmstruktur wurde wie auch in den Vorjahren die EventApp LineUpr ${ }^{2}$ genutzt. Alle Informationen zu den Zeiten, den Räumlichkeiten sowie den Vortragenden finden Vortragende, Teilnehmende und sonstige Interessierte in der ProgrammApp. Tagungsbesucher:innen haben hier die Möglichkeit, sich individuell einen Programmplan zusammenzustellen, und können dies auf dem Smartphone oder dem PC im Responsive Design tagungsbegleitend nutzen ${ }^{3}$.

Gestartet wurde die 23. Jahrestagung am 07. Oktober 2020 mit einer Pre-Konferenz in Form eines virtuellen Barcamps rund um Open Science. ${ }^{4}$ Obschon die Hauptkonferenz zu ca. 2/3 in deutscher Sprache stattfindet, konnte das in Kooperation mit dem Leibniz Forschungsverbund "Open Science ${ }^{\text {"I5 }}$ durchgeführte Barcamp eine vollständig englischsprachige Klientel adressieren.

Ab dem Nachmittag der Vorkonferenz waren Interessent:innen aus Bildung, Politik und Wissenschaft zur Eröffnungsveranstaltung in der HTW Dresden in Präsenz eingeladen. Hier wurde der Erfahrungsaustausch in Form eines Worldcafe umgesetzt. In Summe gab es ca. 50 Online-Teilnehmende im Barcamp und ca. 40 Präsenz-Teilnehmende auf der Vorkonferenz.

Die Hauptkonferenz fand in den Folgetagen vom 08. bis 09. Oktober im Kongresszentrum der DGUV in Dresden statt. Hier wurde im hybriden Format in 2 Workshopräumen zzgl. Foyer und Cateringbereich für die Pausen getagt, mit maximal 50 Teilnehmenden gleichzeitig vor Ort. Das DGUV-Konferenzzentrum bot insgesamt ausreichend Platz für die anteilige Durchführung der Tagung in Präsenz an diesem Ort - unter Beachtung des eigens erstellten Sicherheitskonzeptes.

\footnotetext{
${ }^{4}$ https://www.open-science-conference.eu/barcamp/oscigeneme

5 https://www.leibniz-openscience.de/
}

\footnotetext{
http://www.geneme.de

2 https://lineupr.com/de

${ }^{3}$ https://geneme.lineupr.com/
} 


\section{Die hybride Umsetzung}

Da bereits der Call for Papers während des (ersten) Lockdowns verfasst wurde, stand von Beginn an fest, dass nur ein hybrides, äußerst flexibles Konzept die Durchführung der Konferenz im Herbst 2020 ermöglichen würde. Mindestens das Organisationsteam und die Konferenzleitung sollte in den Räumen vor Ort zusammenkommen können, darüber hinaus sollte die Konferenz notfalls komplett online stattfinden. Vor dem Hintergrund der direkt vor Konferenzbeginn verordneten Beherbergungsverbote und steigender Infektionszahlen hat sich dieses flexible Konzept bewährt, da jede/r Teilnehmende zu jedem Zeitpunkt zwischen Online- und Präsenzteilnahme wechseln konnte.

Als Plattform für die Durchführung wurde MS Teams gewählt, die audiovisuelle Präsentations- und Übertragungstechnik vor Ort wurde durch einen Dienstleister eingerichtet und betreut. Nur durch diese Unterstützung wurde es möglich, dass die Moderatoren vor Ort "in den Raum hinein" für die Präsenzteilnehmenden moderieren konnten, die Sessions gleichzeitig aber auch online in MS Teams zu verfolgen waren. Zudem kam die o.g. Konferenz-App LineUpr für die Planung des individuellen Konferenzprogramms tagungsbegleitend zum Einsatz. ${ }^{6}$

Während der Hauptkonferenz fand die Moderation durch wechselnde Fachexpert:innen durchgehend vor Ort statt. Insgesamt waren die beiden Tage aufgrund der Pandemielage durch unkalkulierbare Anwesenheiten von Beitragenden und Teilnehmenden geprägt.

In Summe gab es ca. 40 Präsenz-Teilnehmende und 60 Online-Teilnehmende auf der Hauptkonferenz. Es wurden hierbei aber durchaus "Misch-Formen“ praktiziert, entweder durch Wechsel des Teilnahmeortes während der beiden Tage, aber auch durch eine Online-Einwahl in die parallele Session aus einem Präsenzsetting heraus. Wichtig für die Möglichkeit eines solch dynamischen Wechsels war die durchgehende Online-Unterstützung, die alle Beiträge und Sessions umfasste.

\section{Konferenzdidaktik}

Da es sich bei der Tagung auch um ein Erprobungsfeld innovativer konferenzdidaktischer Ansätze handelt, stehen für die Vorbereitung und die Durchführung der hybriden Konferenz für die Präsentation und Moderation jeweils neu konfigurierte Handreichungen zur Verfügung (diese sind online abrufbar über die eingebetteten Links):

A) Konferenzdidaktik für die Präsentation

Um der Vielzahl an Beiträgen gerecht zu werden ergaben sich folgende Zeiten entsprechend dem eingereichten Format:

1. Forschungsbeiträge: 20 Minuten (10 min. Input + 10 min. Diskussion)

2. Praxis- und Studierendenbeiträge: $10 \mathrm{Mi}-$ nuten (7 min. Input +3 min. Diskussion)

3. Interaktive Beiträge: 40 Minuten

\section{B) Konferenzdidaktik für die Moderation}

Moderator:innen oblag die Eröffnung und Anleitung der Sessions während der GeNeMe-Tagung. Da diese sowohl in Präsenz als auch online über Microsoft Teams stattfanden, wurden nach Anmeldung zur Tagung personalisierte Zugänge bereitgestellt. In den Sitzungen begrüßten Moderator:innen die Gäste und moderierten deren Beiträge an, orientierten auf die entsprechenden Vortrags- und Diskussionszeiten und zeigten den Referent:innen die verbleibende Redezeit an. Schließlich unterstützten sie die Diskussionsrunden mit anregendem Input und strukturierten Hinweisen zur Ergebnissicherung auf dem virtuellen Whiteboard. Alle Moderator:innen wurden jeweils durch eine studentische Hilfskraft unterstützt. In die Umsetzung waren - soweit zeitlich realisierbar - in systematischer Weise Studierende der Masterstudiengänge "Weiterbildungsforschung und Organisationsentwicklung" und "Wirtschaftswissenschaften" im Rahmen ausgewählter und insofern thematisch passender Lehrveranstaltungen einbezogen.

${ }^{6}$ https://geneme.lineupr.com/geneme2019 


\section{Die Evaluation}

Auf Basis einer während der Tagung online gestützt durchgeführten Befragung aller Teilnehmenden in Form einer freiwilligen und anonymisierten Evaluation konnten einige empirische Befunde erhoben werden.

Unter anderem zeigte die Evaluation (vgl. die nachfolgenden beiden Abbildungen 1 und 2) eine hohe Zufriedenheit sowohl bei in Präsenz als auch bei online Teilnehmenden.

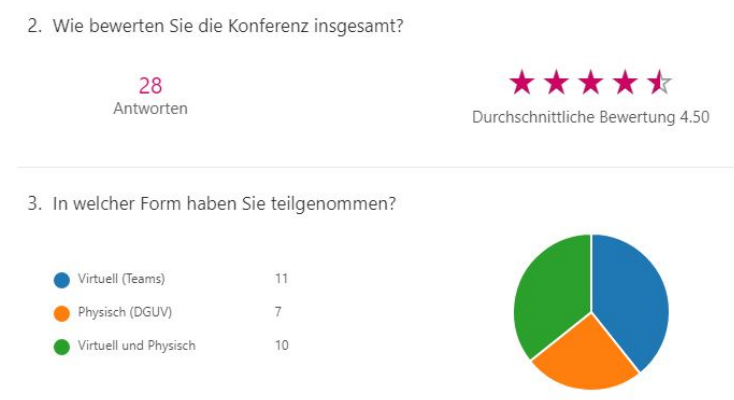

Abb. 1: Konferenzbewertung allgemein

Dabei stimmten 78\% der Befragten der Aussage "Stimmung und Atmosphäre waren sehr gut" voll und ganz zu. Auch wurde die Konferenz im hybriden Format überwiegend (sehr) positiv bewertet, wie die nachfolgende Abbildung 2 zeigt.

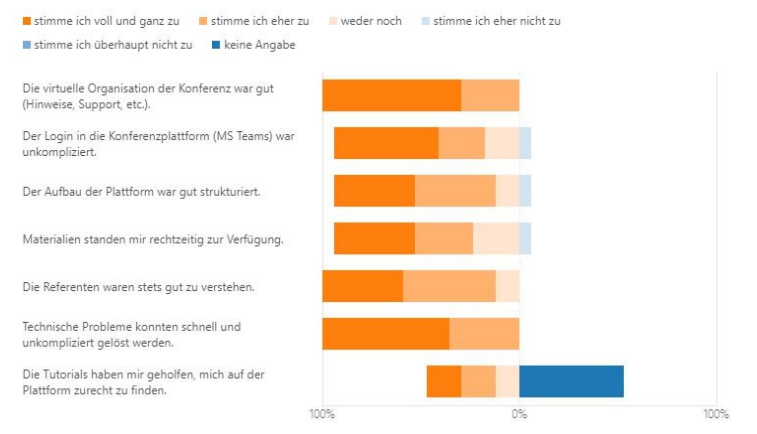

Abb. 2: Konferenzbewertung hybrides Format

\section{Lessons Learned}

Insgesamt stellt die Tagung "Gemeinschaften in Neuen Medien" (GeNeMe) innovative Technologien und Prozesse zur Organisation, Kooperation und Kommunikation in virtuellen Gemeinschaften vor und bildet ein Forum zum fachlichen Austausch, insbesondere auch in den Themenfeldern Wissensmanagement und E-Learning.

Aufgrund der hohen Ansprüche an die Flexibilität der operativen Umsetzung sowie die insgesamt hohe Arbeitsbelastung aller Beteiligten, aber auch die deutlichen Beschränkungen für face-to-face Aktivitäten, musste die traditionell bei der GeNeMe praktizierte didaktische Gestaltung von interaktiven Pausen und Austauschformaten im Jahr 2020 stark reduziert werden. Auch wurden die Pausen infolge des diesmal überaus vollen Programmes gekürzt.

Die Online-Konferenzorganisation mit spezifischen Systemen wird bereits seit längerem als Problemstellung kooperativer Wissensorganisation untersucht [5]. In einem Nicht-Pandemie-Setting mit mehr Planungssicherheit (d.h. auch verbindlicher Buchung von Online- oder Präsenzteilnahme) sollten künftig noch mehr interaktive Formate integriert werden und die Hybridität auch auf diese weiter ausgedehnt werden.

Hier könnten auch verstärkt Socialising-Tools wie wonder.me ${ }^{7}$ für die Pausengestaltung zum Einsatz kommen. Erste Experimente mit hybriden interaktiven Konferenz-Formaten wie hybride Paneldiskussionen sowie ein hybrider Workshop zum Thema Gamification wurden sehr positiv bewertet und werden auch in $\mathrm{Zu}$ kunft in die Konferenz integriert bleiben.

Inwieweit kann man die hier dokumentierten Erfahrungen für die akademische Lehre übernehmen? Einige abschließenden Überlegungen zeigen das bei aller Komplexität vorhandene Potenzial auf:

1. Das hybride Format per se bietet sich sowohl für die Arbeit mit kleinen, als auch groBen Gruppen von Studierenden an. Allerdings besteht im Hinblick zum üblichen Lehrbetrieb ein großer Unterschied weniger in der Digitalität (die gerade Zeiten der Pandemie auch in der Lehre zumeist mittels Videokonferencing funktioniert), sondern vielmehr in der zeitlichen Dimension. Während sich ein Seminar oder eine Vorlesung zumeist über das gesamte Semester erstrecken, wird die Tagung nur für 2-3 Tage durchgeführt. Insofern kommt es zu einer

\footnotetext{
${ }^{7}$ https://www.wonder.me/
} 
nochmals unterschiedlichen Gruppendynamik, die Teilnehmenden kennen sich kaum oder haben zumindest im Kontext der einzelnen Tagungen nicht die Möglichkeit zu einem längerfristigen Beziehungsaufbau, anders als Studierende. Die Tagung wäre insofern eher vergleichbar mit einem Blockseminar.

2. Weiterhin haben wir bei der hybriden Durchführung eine breite Spanne der Situierung der tagungbezogenen Handlungen an einem (teils virtuellen) Tagungsort. Damit stellt sich die Frage nach dem Ansatz der Wirksamkeit der Tagung, der Immersion bei den Teilnehmenden. Dies ist ein Problem, welches einerseits für eine didaktische Situierung typisch ist und insofern auf die Lehre übertragen werden kann.

3. Anderseits fordert dies gerade für den virtuellen Anteil der Tagung die Nutzung entsprechender digital-räumlicher Werkzeuge. Die GeNeMe 2020 hat hier in der Vorbereitungsphase über die zumindest teilweise Nutzung von VR-basierten Kooperationsplattformen wie TriCAT $^{8}$ nachgedacht. Allerdings konnten sich die Ausrichter ob er der Komplexität der Anwendung, aber auch vor dem Hintergrund der nicht unerheblichen Kosten, nicht für eine Nutzung entscheiden. Hier gibt es mit Sicherheit Entwicklungsbedarf. Möglicherweise können ja in 2021 auf der dann 24. GeNeMe Konferenz erstmals Werkzeuge zur VR-basierten Kooperation eingesetzt werden, welche für die akademische Lehre im Portfolio der beteiligten Hochschulen schon etabliert sind.

4. Die GeNeMe nutzt in den drei Phasen der Tagungsvorbereitung, -nachbereitung und durchführung unterschiedliche Social Media Werkzeuge. Dabei handelt es sich um Werkzeuge für Konferenzmanagement (indico) ${ }^{9}$, für die Tagungsdurchführung (MS Teams) und für das Informationsmanagement (LineUpr). Diese Vielfalt völlig unabhängiger Werkzeuge überrascht. Zudem: zumindest an der TU Dresden sind diese Werkzeuge nicht in der Lehre im Einsatz! Warum eigentlich?
Im Ergebnis ist es mehr als wünschenswert, die individuelle Nutzung bzw. den Einsatz digitaler Werkzeuge in wissenschaftlichen Aktivitäten an sich zu unterstützen und zielgerichtet weiterzuentwickeln. Empirische Erkenntnisse zum Stand der Digitalisierung der Wissenschaft in Deutschland, insbes. Sachsen, zeigen hier nach wie vor erheblichen Handlungsbedarf. [6]

\section{Danksagung}

Die Autor:innen danken den Tagungsteilnehmenden für die Beteiligung an der Evaluation sowie den Förderern der Konferenz GeNeMe, insbesondere der GFF. e.V. und dem SMWKT, für die Unterstützung der Veranstaltung im Jahr 2020.

\section{Literatur}

[1] Köhler, T., Schoop, E. \& Kahnwald, N. (2018). The Communities in New Media Conference Series - Research about Knowledge Communities in Business, Science and Public Administration over 20 Years; In Köhler, T., Schoop, E. \& Kahnwald, N. (2018). Communities in New Media. Research on Knowledge Communities in Science, Business, Education \& Public Administration. Proceedings of 21st Conference GeNeMe 2018. Dresden, TUD-Press. http://nbn-re solving.de/urn:nbn:de:bsz:14-qucosa2-334913

[2] Paraskevopoulou, K. \& Köhler, T. (2020). Organizational models in virtual teaching cooperation - documentation and evaluation of organizational didactics in a collaborative higher education project; In: Köhler, T., Schoop, E. \& Kahnwald, N.: Communities in New Media. From hybrid realities to hybrid communities. Proceedings of 23rd Conference GeNeMe; TUDPress, Dresden. https://nbn-resol ving.org/urn:nbn:de:bsz:14-qucosa2-728085

[3] Köhler, T., Neumann, J. \& Lattemann, C. (2021). Organising academia online. Organisation models in elearning versus e-science collaboration; In: Koschtial, C., Köhler, T., Felden, C.: e-Science. Open, social and virtual technology for research collaboration; Progress in IS Series; Berlin, Springer. https://rd.sprin ger.com/chapter/10.1007/978-3-030-66262-2 2

[4] Köhler, T., Schoop, E. \& Kahnwald, N. (2020). Communities in New Media. From hybrid realities to hybrid communities. Proceedings of 23rd Conference GeNeMe 2020. Dresden, TUDPress. https://nbn-re solving.org/urn:nbn:de:bsz:14-qucosa2-728085

[5] Raff, J.-H. \& Köhler, T. (2008). Online-Konferenzorganisationssystem als Problem kooperativer Wissensorganisation: Erfahrungen mit WebEOS beim Kongress der DGfE 2008; DGfE-Mitteilungen, 36 (19).

\footnotetext{
${ }^{9}$ https://getindico.io/

${ }^{8}$ https://www.tricat.net/ 
T. Köhler et al. / Von der Präsenz- zur Hybridveranstaltung

[6] Albrecht, S., Minet, C., Herbst, S., Pscheida, D. \& Köhler, T. (2021). The use of digital tools in scholarly activities. Empirical findings on the state of digitization of science in Germany, with special focus on Saxony; In: Koschtial, C., Köhler, T., Felden, C.: e-Science. Open, social and virtual technology for research collaboration; Progress in IS Series; Berlin, Springer. https://rd.springer.com/chapter/10.1007/978-3-030$\underline{66262-24}$ 